\title{
Sciatic Nerve Injection Palsy in a Dog: Electrodiagnostic Testing and Microsurgical Treatment
}

\author{
Ana Carolina Mortari', Juliany Gomes Quitzan², Claudia Valéria Seullner Brandão² \& Sheila Canevese Rahal²
}

\begin{abstract}
Background: Iatrogenic damage to the ischiatic nerve is considered uncommon and may cause dysfunction with variable clinical signs dependent on type and severity of injury. Due to important role of this nerve in locomotion and weightbearing limb, a poor prognosis for recovery may be observed in many cases. Electromyography analysis may suggest the neuroanatomic localization, diagnosis information, and severity of lesion to determine better therapeutic intervention. Therefore, the aim of this report is to describe the possible cause, diagnosis and treatment of a postinjection ischiatic nerve injury in a dog with complete recovery.

Case: A 3-year-old neutered male dachshund dog was referred to the Veterinary Hospital due to inability to weight support in the right hind limb after diminazene diaceturate intramuscular injection. The gait evaluation showed dropped-hock and knuckling into the digits of the right hind limb and neurologic examination revealed moderate muscle atrophy below to femorotibial joint of the right hind limb with sensory analgesia (superficial and deep) on the lateral, dorsal, and plantar surfaces, absent patellar reflex, and proprioceptive deficit. Electrophysiologic testing was done under general anesthesia in a 2-channel Nicolet Compass Meridian apparatus. Absence of compound muscle action potentials after right fibular and tibial nerve stimulations, and abnormal spontaneous activity in cranial tibial, gastrocnemius and deep digital extensor muscles were observed. A diagnosis of moderate/severe axonotmesis of sciatic nerve was achieved. Under microscope magnification, all adherent adjacent tissue and epineural sheat were removed. Due this, a small epineural window was created. On neurological examination performed 30 days after surgery, complete recovery of sensitivity of the right hind limb, and normal proprioception were observed. The muscle atrophy was also noted to have improved.

Discussion: The ischiatic nerve mechanisms of injury include direct needle trauma, the drug or vehicle used for injection, or secondary constriction by scar, factors that may be associated to damage nerve observed in the present case. During a sciatic nerve injection, the combination of intrafascicular placement of a needle and high-pressure injection may cause severe fascicular damage and persistent neurologic deficits. In the present case, damage to the nerve probably was not caused by the injection needle, but due to injection agent. Chemical irritation or toxic reaction to the agent may cause different degrees of nerve injury. The electrophysiologic testing is an important tool for determining alteration of function and integrity of the axonal motor unit. In the present report, the electrophysiologic testing showed denervation potentials in the muscles innervated by the sciatic nerve (positive waves and fibrillation potentials), and the absence of compound muscle action potentials was indicative of severe axonal damage of the right ischiatic nerve. In human patients with postinjection ischiatic nerve injury, early surgical treatment with neurolysis or resection and anastomosis are the procedures recommended. In the present report, external neurolysis and epineural window were used showing excellent functional results. The epineural window was performed due to adherence of tissue and scar surrounding the nerve, permitting neural decompression.
\end{abstract}

Keywords: axonotmesis, neurophysiology, neurolysis, dogs. 


\section{INTRODUCTION}

Peripheral nerve injuries are common in dogs and cats and may cause temporary or permanent neurological damage $[1,5,8,10]$. However, iatrogenic damage to the ischiatic nerve is considered uncommon and cause dysfunction with variable clinical signs dependent on type and severity of injury [5]. Due to important role of this nerve in locomotion and weight-bearing limb, a poor prognosis for recovery may be observed in many cases [2]. Compound motor action potential amplitude in nerve conduction studies reflects the numbers of muscle fibers that depolarize. In traumatic nerve injuries, low amplitudes most often result from loss axons [12,15,17]. In these cases, the presence of abnormal spontaneous activity on electromyography analysis may suggest the neuroanatomic localization, diagnosis information and severity of lesion $[3,12,17]$ to determine better therapeutic intervention.

Therefore, the aim of this report is to describe the possible cause, diagnosis and treatment of a postinjection ischiatic nerve injury in a dog with complete recovery.

\section{CASE}

A 3-year-old neutered male dachshund dog was referred to the Veterinary Hospital due to inability to weight support in the right hind limb. According to the owner, the clinical signs were present immediately after diminazene diaceturate and antipyrine have been injected intramuscularly in the gluteal region because of babesiosis. After this, the dog had been treated unsuccessfully with vitamin B complex and acupuncture for approximately 20 days.

The gait evaluation showed dropped-hock and knuckling into the digits of the right hind limb (Figure 1a). Neurologic examination revealed moderate muscle atrophy below to femorotibial joint of the right hind limb with sensory analgesia (superficial and deep) on the lateral, dorsal, and plantar surfaces, absent patellar reflex, and proprioceptive deficit. All cranial nerve reflexes were normal.

Electrophysiologic testing was done under general anesthesia. After premedication with acepromazine ${ }^{1}$ $0.2 \%[0.05 \mathrm{mg} / \mathrm{kg}$ bodyweight (BW), IM], and morphi$\mathrm{ne}^{1}(0.5 \mathrm{mg} / \mathrm{kg} \mathrm{BW}, \mathrm{IM})$, general anesthesia was induced with propofol ${ }^{1}$ (4 mg/kg BW, IV), and maintained with isoflurane. Motor nerve conduction and electromyography were performed in a 2-channel Nicolet Compass Meridian apparatus ${ }^{2}$ using differential amplifiers and common-mode rejection ratios of approximately $90 \mathrm{~dB}$. Band-pass filter was set to $20-3,000 \mathrm{~Hz}$, sensibility to 1 or $2 \mathrm{mV} / \mathrm{cm}$, and analysis time to $2 \mathrm{~ms} / \mathrm{cm}$. In both hind limbs, motor nerve conduction studies (MNCS) were obtained from the sciatic-tibial nerves using surface electrodes. Proximal stimulation site was just caudal to the greater trochanter and distal stimulation site was on the lateral and caudal aspect of the distal tibial third after clipping and cleaning the skin. Absence of compound muscle action potentials were observed after right fibular and tibial nerve stimulations. Increase of insertional activity and abnormal spontaneous activity included positive waves and fibrillation potentials were observed in cranial tibial, gastrocnemius (Figure 1b) and deep digital extensor muscles. Normal rest activity was observed in semitendinosus and semimembranosus muscles. A diagnosis of moderate/severe axonotmesis of sciatic nerve at the level of the right stifle joint was achieved based on the results of the aforementioned tests.

Surgical procedure to explore injured nerve was performed. A caudolateral approach was used at the level of distal femur. The biceps femoris muscle was retracted caudally and the vastus lateralis muscle was retracted cranially to expose the sciatic nerve. A yellowish color material with a jelly-like aspect and fibrous tissue was surrounding the sciatic nerve proximal to bifurcation into the common fibular and the tibial nerves, above the gastrocnemius muscle. Under microscope magnification, all adherent adjacent tissue and epineural sheat were removed. Due this, a small epineural window was created.

On neurological examination performed 30 days after surgery, it was observed complete recovery of sensitivity of the right hind limb, and normal proprioception. The muscle atrophy was also noted to have improved (Figure 1c).

\section{DISCUSSION}

The ischiatic nerve is located deep to the muscle mass, that protected it against injuries [2]. In dogs, the ischiatic nerve injuries have been associated with pelvic fracture, surgical exploration of the ilium wing, acetabulum, sacroiliac joint and caudal proximal femoral region, and perineal herniorrhaphy $[5,8]$. Other mechanisms of injury include direct needle trauma, the drug or vehicle used for injection, or secondary constriction by scar $[5,17]$, factors that may be associated to damage nerve observed in the present case. 


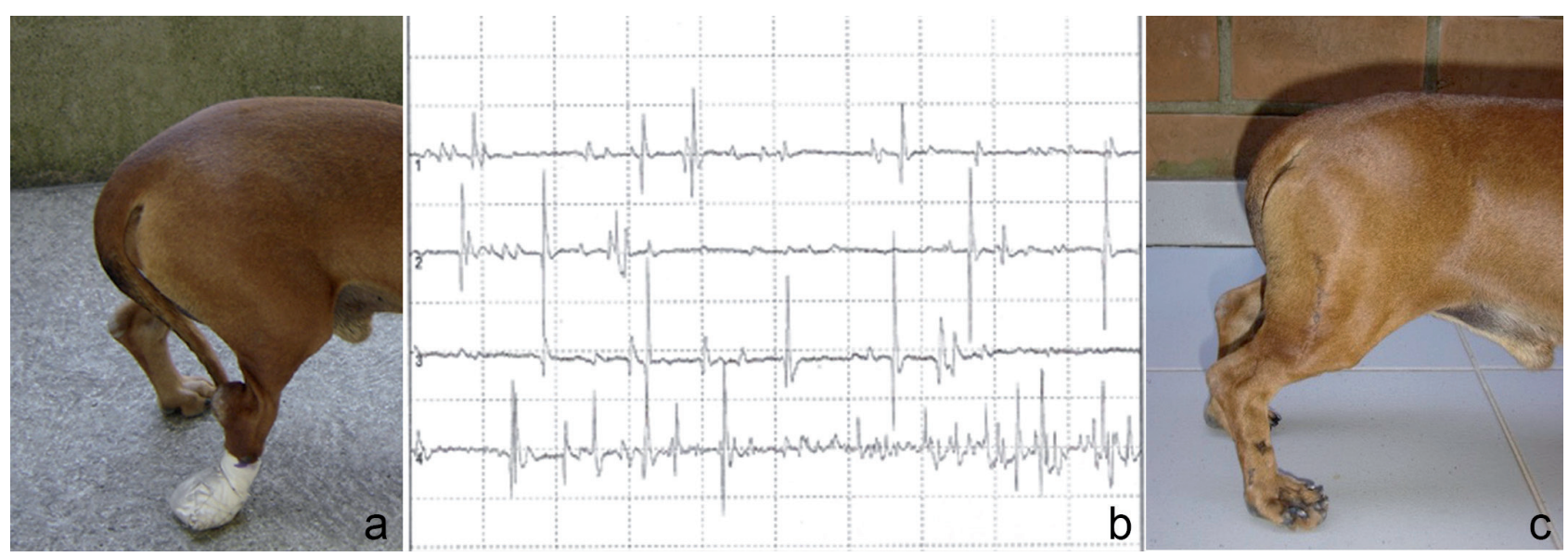

Figure 1. a- Monoplegia, proprioceptive deficit and muscular atrophy on distal right hind limb; b- Abnormal spontaneous activity - positive waves and fibrillation potentials were observed in gastrocnemius muscle; c- Neurological examination of the dog 30 days after surgery showing normal weight support and no muscle atrophy of the right hind limb.

The combination of intrafascicular placement of a needle and high-pressure injection may cause severe fascicular damage and persistent neurologic deficits [9]. In addition, depending on site, agent and volume injected, a mixed injury with severe injury lesion to one fascicle or relative sparing of adjacent fascicles can be observed [7]. In the present case, nerve disruption and perineural fibrosis surrounding the ischiatic nerve were not recognized intraoperatively. Thus, damage to the nerve probably was not caused by the injection needle, but due to injection agent.

Chemical irritation or toxic reaction to the agent may cause different degrees of nerve injury [4,5]. Diminazene diaceturate and antipyrine were probably the chemical agents responsible for the injury in this report. Although diminazene aceturate is recommended to dogs with babesiosis, side effects such as nervous signs, anaphylaxis and vomiting have been reported $[11,14]$. However, none of the dogs showed any pain reaction at the time of injection and no muscle swelling was seen at the injection site [11, 13-14].

In spite of the clinical signs, the electrophysiologic testing is an important tool for determining alteration of function and integrity of the axonal motor unit [2]. In the present study, the electrophysiologic testing showed abnormal spontaneous activity in different muscles innervated by ischiatic nerve [2,15]. Besides the wave morphology (positive sharp waves and fibrillation potentials) has suggested denervation potentials, the absence of compound muscle action potentials was indicative of severe axonal damage of the right ischiatic nerve. In general, positive sharp waves and fibrillation potentials are observed two or three weeks after axonotmesis [3,6,12] as observed in the present study.

In human patients with postinjection ischiatic nerve injury, early surgical treatment is considered a better chance of a full recovery and to decrease the development of long-term sequelae [17-18]. The neurolysis or resection and anastomosis are the procedures recommended [18]. A sciatic nerve injury after intramuscular injection in a dog was treated by implantation of autologous contralateral tibial nerve autograft resulting in good functional recovery, but strength deficits was present [5]. In the present report, external neurolysis and epineural window were used showing excellent functional results. The epineural window was performed due to adherence of tissue and scar surrounding the nerve.

Therefore, this case report suggests that intramuscular diminazene diaceturate and antipyrine injection may cause ischiatic nerve injury. The positive outcome of the axonotmesis in this case was attributed to surgical procedures of neurolysis and epineural window.

\section{MANUFACTURERS}

${ }^{1}$ Laboratório Cristália. São Paulo, SP, Brazil.

${ }^{2}$ Nicolet Biomedical Inc. Madison, WI, USA.

Declaration of interest. The authors report no conflicts of interest. The authors alone are responsible for the content and writing of the paper. 


\section{REFERENCES}

1 Chambers J.N. \& Hardie E.M. 1986. Localization and management of sciatic nerve injury due to ischial or acetabular fracture. Journal American Animal Hospital Association. 22: 539-544.

2 DeLahunta A., Glass E. \& Kent M. 2015. Lower Motor Neuron: Spinal Nerve, General Somatic Efferent System. In: De Lahunta A., Glass E. \& Kent M. (Eds). Veterinary Neuroanatomy and Clinical Neurology. 4th edn. Philadelphia: Saunders, pp.102-161.

3 Dumitru D., Zwarts M. \& Amato A.A. 2002. Peripheral nervous system's reaction to injury. In: Dumitru D., Amato A.A. \& Zwarts M. (Eds). Electrodiagnostic Medicine. 2nd edn. Philadelphia: Hanley \& Belfus, pp.115-158.

4 Esquenazi Y., Park S.H., Kline D.G. \& Kim D.H. 2016. Surgical management and outcome of iatrogenic radial nerve injection injuries. Clinical Neurology and Neurosurgery. 142: 98-103.

5 Forterre F., Tomek A., Rytz U., Brunnberg L., Jaggy A. \& Spreng D. 2007. Iatrogenic sciatic nerve injury in eighteen dogs and nine cats (1997-2006). Veterinary Surgery. 36: 464-471.

6 Gemmmil T. \& Mckee M. 2012. Monoparesis and neurological causes of lameness. In: Platt S. \& Garosi L. (Eds). Small Animal Neurological Emergencies. Boca Raton: Taylor \& Francis Group, pp.299-316.

7 Gentili F., Hudson A., Kline D. \& Hunter D. 1980. Early changes following injection injury of peripheral nerves. Canadian Journal of Surgery. 23(2): 177-182.

8 Gilmore D.R. 1984. Sciatic nerve injury in twenty-nine dogs. Journal American Animal Hospital Association. 20(3): 403-407.

9 Hadzic A., Dilberovic F., Shah S., Kulenovic A., Kapur E., Zaciragic A., Cosovic E., Vuckovic I., Divanovic K.A., Mornjakovic Z., Thys D.M. \& Santos A.C. 2004. Combination of intraneural injection and high injection pressure leads to fascicular injury and neurologic deficits in dogs. Regional Anesthesia and Pain Medicine. 29(5): 417-423.

10 Jacobson A. \& Schrader S.C. 1987. Peripheral nerve injury associated with fracture or fracture-dislocation of the pelvis in dogs and cats: 34 cases (1978-1982). Journal American Veterinary Medical Association. 190(5): 569-572.

11 Joubert K.E., Kettner F., Lobetti R.G. \& Miller D.M. 2003. The effects of diminazene aceturate on systemic blood pressure in clinically healthy adult dogs. Journal of the South African Veterinary Association. 74(3): 69-71.

12 Kimura J. 2013. Anatomy and physiology of the peripheral nerve and type of nerve pathology. In: Kimura J. (Ed). Electrodiagnosis in Diseases of Nerve and Muscle: Principles and Practice. 4th edn. New York: Oxford University Press, pp.49-73.

13 Lin M.Y. \& Huang H.P. 2010. Use of a doxycycline-enrofloxacin-metronidazole combination with/without diminazene diaceturate to treat naturally occurring canine babesiosis caused by Babesia gibsoni. Acta Veterinaria Scandinavica. 52(1): 27.

14 Miller D.M., Swan G.E. \& Lobetti R.G. 2005. The pharmacokinetics of diminazene aceturate after intramuscular administration in healthy dogs. Journal of the South African Veterinary Association. 76(3): 146-150.

15 Preston D.C. \& Shapiro B.E. 2005. Basic electromyography: analysis of spontaneous activity. In: Preston D.C. \& Shapiro B.E. (Eds). Electromyography and Neuromuscular Disorders. Clinical - Electrophysiologic Correlations. 2nd edn. Philadelphia: Elsevier, pp.199-214.

16 Senes F.M., Campus R., Becchetti F. \& Catena N. 2009. Sciatic nerve injection palsy in the child: early microsurgical treatment and long-term results. Microsurgery. 29(6): 443-448.

17 Topuz K., Kutlay M., Simsek H., Atabey C., Demircan M. \& Senol G.M. 2011. Early surgical treatment protocol for sciatic nerve injury due to injection: a retrospective study. British Journal of Neurosurgery. 25(4): 509-515.

18 Villarejo F.J. \& Pascual A.M. 1993. Injection injury of the sciatic nerve (370 cases). Child's nervous system. 9(4): 229-232. 